KFA-IKP(Th)-1994-17

20 May 1994

\title{
Scanning the BFKL pomeron in elastic production of vector mesons at HERA
}

\author{
J.Nemchik ${ }^{a, b}$, N.N. Nikolaev ${ }^{b, c}$, and B.G. Zakharov ${ }^{c}$ \\ ${ }^{a}$ Institute of Experimental Physics, Slovak Academy of Sciences, \\ Watsonova 47, 04353 Kosice, Slovak Republik \\ ${ }^{b} I K P$ (Theorie), KFA Jülich, 5170 Jülich, Germany \\ ${ }^{c}$ L. D. Landau Institute for Theoretical Physics, GSP-1, 117940, \\ ul. Kosygina 2, Moscow 117334, Russia.
}

\begin{abstract}
Elastic production of vector mesons $\gamma^{*} N \rightarrow V N$ is the pomeron-exchange dominated diffractive reaction with much potential of probing the BFKL pomeron. The BFKL pomeron can conveniently be described in terms of the dipole cross section which is a solution of the generalized BFKL equation. In this paper we discuss, how the energy and $Q^{2}$ dependence of elastic production of vector mesons at HERA will allow scanning the dipole cross section as a function of dipole size $r$. We show that determinaton of the intercept of the BFKL pomeron requires measuring the $\rho^{0}$ and $J / \Psi$ production at $Q^{2} \sim(100-200) \mathrm{GeV}^{2}$ and/or the quasireal photoproduction of the $\Upsilon$. We present predictions for the effective intercept in the kinematic range of the forthcoming HERA experiments, which can shed much light on the nonperturbative component of the pomeron.
\end{abstract}

E-mail: kph154@zam001.zam.kfa-juelich.de 
Elastic real and virtual photoproduction of vector mesons

$$
\gamma^{*} p \rightarrow V p, \quad V=\rho^{0}, \omega^{0}, \phi^{0}, J / \Psi, \Upsilon \ldots
$$

is the typical diffractive reaction, dominated by pomeron exchange. Determination of parameters of the BFKL pomeron [1], in particular of its intercept $\Delta_{\mathbb{P}}$, is an outstanding problem for the HERA experiments on small $x$, and exploring the potential of reaction (1) is of great importance. In [2-4] we developed a novel approach to the BFKL pomeron in terms of the dipole cross section $\sigma(\xi, r)$ which satisfies the generalized BFKL equation. Here $r$ is the size of the color dipole, $\xi=\log \left[2 m_{p} \nu /\left(Q^{2}+m_{V}^{2}\right)\right]$ is the rapidity, $m_{V}$ is the vector meson mass, $Q^{2}$ is the virtuality of the photon and $\nu$ its energy in the proton rest frame. At very high $\nu$ and/or very small Bjorken variable $x$ one expects

$$
\sigma(\xi, r)=\sigma_{\mathbf{I P}}(r) \exp \left(\Delta_{\mathbf{I P}} \xi\right)
$$

and

$$
F_{2}\left(x, Q^{2}\right) \propto \exp \left(\Delta_{\mathbf{I P}} \xi\right) \sim\left(\frac{1}{x}\right)^{\Delta_{\mathbf{I}}}
$$

with the intercept $\Delta_{\mathbb{P}}$ which does not depend on $Q^{2}$, the real photoproduction $Q^{2}=0$ not excepted. Understanding the onset of the BFKL regime (2, 3) is the most pressing issue, because experimentally a steep rise of $F_{2}\left(x, Q^{2}\right)$ at HERA [5] is accompanied by a much slower rise of the real photoabsorption cross section [6].

What makes reactions (1) exceptionally important is the scanning phenomenon discovered in [7-11], by which elastic production amplitude probes the dipole cross section at the scanning radius

$$
r_{S} \approx \frac{C}{\sqrt{m_{V}^{2}+Q^{2}}} .
$$

The scale parameter $C$ is rather large, and in [11] we gave an estimate $C \approx 6$. Changing $Q^{2}$ and the mass of the produced vector meson, one can probe $\sigma(\xi, r)$ and measure the effective intercept $\Delta_{e f f}(\xi, r)=\partial \log \sigma(\xi, r) / \partial \xi$ in a very broad range of dipole size $r$, from the nonperturbative regime of $r \gtrsim 1 \mathrm{f}$ down to the perturbative regime of $r \ll 1 \mathrm{f}$. The HERA experiments are already amassing the data on production of vector mesons, and the purpose of this communication is to present the detailed predictions for reactions (四) from the approach to the BFKL pomeron developed in [2-4,12-15]. 
In order to set up the framework, we briefly review properties of the dipole cross section. In $[3,4,12,13]$ we studied solutions of the generalized BFKL equation in the realistic model with the finite correlation radius $R_{c}$ for perturbative gluons and the running QCD coupling $\alpha_{S}(r)$, which freezes at large distances $\alpha_{S}\left(r \geq R_{f}\right)=\alpha_{S}^{(f r)}=0.8$. With the $R_{c}=0.3 f$ as suggested by the lattice QCD studies, $\Delta_{\mathbb{P}}=0.4[4,12]$. The major findings are:

Firstly, the onset of the asymptotic behaviour (2, 30) was found to be extremely slow. Namely, the effective intercept $\Delta_{e f f}(\xi, r)$ varies quite strongly in the range of $r$ and $\xi$ relevant to the HERA experiments on DIS. The usually discussed structure function $F_{2}\left(x, Q^{2}\right)$ receives contributions from a broad range of $r$ and, for this reason, its $x$ dependence does not allow a realiable experimental determination of the intercept $\Delta_{\mathbb{P}}$.

Secondly, by the diffusion property of the BFKL kernel $[1,4,12]$, at asymptotic energies the behaviour (2) takes over at all values of $r$, including $r>R_{c}$, thus unifying the asymptotic energy dependence of the bare pomeron-exchange contribution to hadronic scattering, the real photoproduction and deep inelastic scattering. Furthermore, by the same diffusion property, the intercept $\Delta_{\mathbb{I P}}$ is almost entirely controlled by the region of $r \sim R_{c}[4,12]$.

Thirdly, there happens to exist a "magic" size $r_{\Delta} \approx \frac{1}{2} R_{c}$, at which the precocious asymptotic behaviour $\Delta_{e f f}(\xi, r) \approx \Delta_{\mathbb{P}}$ will persist over the whole range of $x$ at HERA. In $[4,15]$ we showed how one can zoom at $r \sim r_{\Delta}$, measuring at HERA the charm structure function at $Q^{2} \lesssim 10 \mathrm{GeV}^{2}$, the longitudinal structure function $F_{L}\left(x, Q^{2}\right)$ at $Q^{2} \sim(10-$ 40) $\mathrm{GeV}^{2}$ and the scaling violations $\partial F_{T}\left(x, Q^{2}\right) / \partial \log Q^{2}$ at $Q^{2} \sim(2-10) \mathrm{GeV}^{2}$.

Fourthly, the BFKL pomeron describes the exchange by perturbative gluons with the typical interaction radius $\sim R_{c}$. At low energy and large dipole size $r \gg R_{c}$, the nonperturbative scattering mechanism can take over. The rise of the BFKL perturbative cross section is driven by the rising multiplicity of perturbative gluons in the high-energy photon (hadron), for the nonperturbative mechanism one can expect the (approximately) energyindependent contribution $\sigma^{(n p t)}(r)$ to the dipole cross section. A large contribution from the $\sigma^{(n p t)}(r)$ reconciles the steep rise of $F_{2}\left(x, Q^{2}\right)[5]$ with a much weaker energy dependence of the real photoabsorption cross section [6]. As we shall discuss below, the scanning phenomenon allows to study in much detail a transition between the nonperturbative and 
perturbative regimes, which makes reactions (1) particularly important.

The origin of the scanning phenomenon is as follows: In the dipole-cross section representation, the amplitude of the forward production $\gamma^{*} p \rightarrow V N$ of the (T) transverse and (L) longitudinally polarized vector mesons reads [7-11,16,17]

$$
\begin{gathered}
M_{T}\left(\xi, Q^{2}\right)=\frac{N_{c} C_{V} \sqrt{4 \pi \alpha_{e m}}}{(2 \pi)^{2}} . \\
=\frac{1}{\left(m_{V}^{2}+Q^{2}\right)^{2}} \int \frac{d r^{2}}{r^{2}} \frac{\sigma(\xi, r)}{r^{2}} W_{T}\left(Q^{2}, r^{2}\right) \\
M_{L}\left(\xi, Q^{2}\right)=\frac{N_{c} C_{V} \sqrt{4 \pi \alpha_{e m}}}{(2 \pi)^{2}} \frac{2 \sqrt{Q^{2}}}{m_{V}} . \\
\cdot \int d^{2} \vec{r} \sigma(\xi, r) \int_{0}^{1} d z\left\{\left[m_{q}^{2}+z(1-z) m_{V}^{2}\right] K_{0}(\varepsilon r) \phi(r, z)-\varepsilon K_{1}(\varepsilon r) \partial_{r} \phi(z, r)\right\} \\
=\frac{1}{\left(m_{V}^{2}+Q^{2}\right)^{2}} \frac{2 \sqrt{Q^{2}}}{m_{V}} \int \frac{d r^{2}}{r^{2}} \frac{\sigma(\xi, r)}{r^{2}} W_{L}\left(Q^{2}, r^{2}\right)
\end{gathered}
$$

Here $N_{c}=3$ is the number of colours, $C_{V}=\frac{1}{\sqrt{2}}, \frac{1}{3 \sqrt{2}}, \frac{1}{3}, \frac{2}{3}$ for the $\rho^{0}, \omega_{0}, \phi^{0}, J / \Psi$ production, respectively, $K_{0,1}(x)$ is the modified Bessel function,

$$
\varepsilon^{2}=m_{q}^{2}+z(1-z) Q^{2}
$$

$\phi(z, r)$ is the lightcone wave function of the $q \bar{q}$ Fock state of the vector meson in the mixed $(z, \vec{r})$-representation, where $\vec{r}$ is the transverse separation of the quark and antiquark and $z$ is a fraction of the lightcone momentum carried by the quark. The normalization is such that

$$
\frac{N_{c}}{2 \pi} \int d^{2} \vec{r} \int_{0}^{1} \frac{d z}{z(1-z)}\left\{m_{q}^{2} \phi^{2}(r, z)+\left[z^{2}+(1-z)^{2}\right]\left[\partial_{r} \phi(r, z)\right]^{2}\right\}=1 .
$$

The dipole cross section can be related to the gluon structure function of the target proton $G\left(x, q^{2}\right)[2,17,18]$

$$
\sigma(\xi, r)=\frac{\pi^{2}}{3} r^{2} \alpha_{S}(r) G\left(x=\exp (\xi), q^{2}=\frac{A}{r^{2}}\right) \propto r^{2}\left[\frac{1}{\alpha_{S}(r)}\right]^{\gamma-1} \exp \left(\Delta_{\mathbb{P}} \xi\right),
$$

where $\gamma=4 / 3 \Delta_{\mathbf{I P}}[14]$ and $A \approx 10$ (the emergence of this large numerical factor is explained in [13]). The most important property of the dipole cross section is the color transparency driven dependence $\propto r^{2}$ at small $r$. Because $K_{0,1}(x) \propto \exp (-x)$ at large $x$, and the wave 
function of the vector meson is smooth, the amplitudes (55,6) will be dominated by the contribution from $r \approx 3 / \varepsilon$, which in the nonrelativistic approximation of $m_{V} \sim 2 m_{q}$ and $z \sim \frac{1}{2}$ leads to the scanning radius (四) and the estimate $C \approx 6$. When the scanning radius is small, $r_{S} \lesssim R_{V}$, where $R_{V}$ is the vector meson radius, the amplitudes (6., (1) can be evaluated as

$$
\begin{gathered}
M_{T} \propto r_{S}^{2} \sigma\left(\xi, r_{S}\right) \propto \frac{1}{\left(m_{V}^{2}+Q^{2}\right)^{2}}, \\
M_{L} \propto \frac{\sqrt{Q^{2}}}{m_{V}} r_{S}^{2} \sigma\left(\xi, r_{S}\right) \propto \frac{\sqrt{Q^{2}}}{m_{V}} \frac{1}{\left(m_{V}^{2}+Q^{2}\right)^{2}} \propto \frac{\sqrt{Q^{2}}}{m_{V}} M_{T},
\end{gathered}
$$

which must be contrasted to prediction of the vector dominance model $M_{T} \propto 1 /\left(m_{V}^{2}+Q^{2}\right)$. This departure from the vector dominance comes entirely from colour transparency property of the dipole cross section [11]. Production of the longitudinally polarized vector mesons dominates at $Q^{2} \gg m_{V}^{2}$.

The scanning property is quantified by weight function $W_{T, L}\left(Q^{2}, r^{2}\right)$ in Eqs. (5.6), which we introduced following [13]. The $W_{T, L}\left(Q^{2}, r^{2}\right)$ are sharply peaked (Fig. 1) and, because $\sigma(\xi, r) / r^{2}$ is a slow function of $r$, see Eq. (9), the position of the peak gives the scanning radius $r_{S}$. At sufficiently large $Q^{2}$ such that $r_{S} \lesssim R_{V}$, which typically means $Q^{2} \gg m_{V}^{2}$, the position of the peak in the variable $y=r^{2}\left(Q^{2}+m_{V}^{2}\right)$ very weakly depends on $Q^{2}$. For production of the longitudinally polarized vector mesons, the peak corresponds to $C_{L}(J / \Psi) \sim 7$ and $C_{L}\left(\rho^{0}\right) \sim 8$. For the transversely polarized $\rho^{0}$ mesons, with rising $Q^{2}$ the peak is slowly drifting towards $C_{T} \sim 12$ at $Q^{2} \sim 100 \mathrm{GeV}^{2}$, which can be understood as follows [11]: At a fixed $z$, the scanning radius $r_{S} \approx 3 / \varepsilon$. For the longitudinal photons, the wave function is peaked at $z \sim \frac{1}{2}$, hence the estimate $C \approx 6$ is rather accurate (for the related discussion of the longitudinal structure function see $[2,17,20])$. In the opposite to that, the transverse structure function receives significant contribution from the very asymmetric pairs with $z,(1-z) \sim m_{q}^{2} / Q^{2}$, which have a large, hadronic transverse size $r \sim 1 / m_{q}[2,17]$. Because of the contribution of such asymmetric pairs, the effective scanning radius for the transversely polarized vector mesons decreases with $Q^{2}$ less rapidly than given by the simple estimate (東). The results shown in Fig. 1, were obtained with the relativized wave function of vector mesons, which has the correct short distance behaviour driven by hard gluon exchange [16]. Notice, that because of very large numerical value of $C_{T, L}$, the scanning radius 
$r_{S}$ Eq. (1) remains large, and the relativistic effects in wave functions are still marginal, in a very broad range of $Q^{2}$. For instance, for the $J / \Psi$ we find $C_{T} \approx C_{L}$ and very close to the nonrelativistic estimate $C_{T, L} \approx 6$ even at $Q^{2} \gg m_{V}^{2}$.

At small $Q^{2}$, the scanning radius $r_{S}$ is large, and one probes the dipole cross section in the nonperturbative domain of $r$. In Fig. 2 we present the decomposition of the dipole cross section into the nonperturbative component $\sigma^{(n p t)}(r)$ and the $\sigma^{(p t)}(\xi, r)$ which is a solution of the perturbative BFKL equation日:

$$
\sigma(\xi, r)=\sigma^{(n p t)}(r)+\sigma^{(p t)}(\xi, r)
$$

This additivity of the bare pomeron cross sections and/or of the eikonal functions when the unitarization is considered, is the simplest assumption, and more refined treatment will be necessary in an analysis of the future high-precision data. The shape of the nonperturbative cross section is the largest unknown in the problem, and is mostly driven by our analysis of the $Q^{2}$ dependence of the photoproduction of vector mesons [8-11] and of structure functions at moderate $Q^{2}$ [18], which are well described by the dipole cross section calculated in [17]. Therefore, the $\sigma^{(n p t)}(r)$ is tuned so as to reproduce the dipole cross section of ref. [17] at $\xi=\xi_{0}=-\log 0.03$, which we take as the starting point for the BFKL evolution. In Fig. 2 we show how the $\sigma^{(p t)}(\xi, r)$ evolves with the rapidity $\xi$. At small $r$, the total dipole cross section is dominated by the BFKL cross section $\sigma^{(p t)}(\xi, r)$. In this region, because of the so-called double logarithmic effects, the effective intercept $\Delta_{e f f}(\xi, r)>\Delta_{\mathbb{I P}}[3,4]$. At large $r$, $\Delta_{e f f}(\xi, r) \ll \Delta_{\mathbb{P}}$ because of the large contribution from the nonperturbative cross section $\sigma^{(n p t)}(r)$. The analysis $[4,13]$ has shown that there exists a magic radius $r \sim r_{\Delta}$, at which $\Delta_{e f f}(\xi, r) \approx \Delta_{\mathbb{P}}$ starting already at moderate $\xi$. At this value of $r$, the nonperturbative cross section is small, and zooming at $r \sim r_{\Delta}$ shall allow direct measurement of $\Delta_{\mathbb{I P}}$ at HERA.

The above discussion refers to the bare pomeron cross section. With the conventional Gaussian parametrization of the elastic scattering peak, $d \sigma_{e l} / d t \propto \exp (-B|t|)$, where $|t|$ is

\footnotetext{
${ }^{1}$ This Fig. 2 updates Fig. 1 of Ref. [14], in which the mistake was made in plotting $\sigma^{(p t)}(\xi, r)$. This plotting mistake does not affect any of predictions made in [14], though. Our choice of $\sigma^{(n p t)}(r)$ in this paper is slightly different from that in [14].
} 
the momentum transfer squared, the profile function of elastic scattering takes the form

$$
\Gamma(b)=\frac{\sigma_{t o t}}{4 \pi B} \exp \left(-\frac{b^{2}}{2 B}\right),
$$

where $b$ is the impact parameter. The rise of the bare pomeron cross section will eventually conflict the $s$-channel unitarity bound $\Gamma(b) \leq 1$. Unique solution of the unitarization problem is lacking; for the crude estimate we apply the $\mathcal{K}$-matrix unitraization

$$
\Gamma(b)=\frac{\Gamma_{0}(b)}{1+\Gamma_{0}(b)} .
$$

When the rising strength of the bare pomeron interaction $\Gamma_{0}(b) \gg 1$, the unitraized profile function tends to the black disc limit $\Gamma(b) \rightarrow 1$. The $\mathcal{K}$-matrix unitarization leads to a particularly simple form of the unitarized total cross section [19]

$$
\sigma^{(U)}(\xi, r)=4 \pi B \log \left(1+\frac{\sigma(\xi, r)}{4 \pi B}\right) .
$$

For a crude evaluation of the unitarization effects, we take the energy-independent diffraction slope $B=10 \mathrm{GeV}^{-2}$. Evidently, the unitarization effects only become important when $\sigma(\xi, r) \gtrsim 4 \pi B \sim 50 \mathrm{mb}$, i.e., either at large $r$ and/or at very high energy. As we shall see below, in the kinematical range of the HERA experiments, unitarization effects are marginal.

Our predictions for the energy dependence of different photoproduction observables are shown in Fig. 3,4. In Fig. 3 we show the real photoabsorption cross section. It was calculated using the wave function of the photon derived in [17], assuming the effective quark mass $m_{u, d}=0.15 \mathrm{GeV}$, which is the sole parameter in the quark wave function. Only the nonperturbative contribution to $\sigma_{\text {tot }}(\gamma p)$ is sensitive to this parameter. For the comparison purposes, we also show our predictions for $\sigma_{\text {tot }}\left(\rho^{0} p\right)$, which is close to $\sigma_{t o t}(\pi N)$ and has a rise consistent with the observed trend of the hadronic total cross sections (for a recent review and high-energy extrapolations see [22]). We attribute the slow rise of $\sigma_{t o t}(\gamma p)$ and $\sigma_{\text {tot }}\left(\rho^{0} p\right)$ to the large contribution from the nonperturbative large-size cross section (for the early discussion of such a scenario see [23]). The effect of the unitarization slowly rises with energy. It predominantly affects the nonperturbative cross section and, given the uncertanty in the absolute normalization of the $\sigma^{(n p t)}(r)$, at small energies it can be compensated for by upwards renormalization of the input $\sigma^{(n p t)}(r)$. The energy dependent part of the 
unitarization correction is, however, the genuine effect and brings our results for $\sigma_{\text {tot }}(\gamma p)$ to a better agreement with the experiment [6]. The simple $\mathcal{K}$-matrix unitarization, as well as the eikonal unitarization, lacks the so-called triple-pomeron contribution, which enhances the effect of unitarization at high energies $([2,19]$ and references therein) and can tame the somewhat too rapid a growth of $\sigma_{t o t}(\gamma p)$, a description of the low energy data [21] also can be augmented, by adding to the nonperturbative cross-section the Regge-behaving $\propto 1 / \sqrt{\nu}$ terms [22]. However, the purpose of the present analysis is understanding the gross features of the BFKL phenomenology rather than fitting the low- $Q^{2}$ experimental data. Recently, there was much discussion on the perturbative QCD contribution to the real photoabsorption cross section $\sigma_{t o t}(\gamma p)$, in which the eikonal is evaluated with the BFKL cross section substituted for the inclusive minijet cross section ([24] and references therein) which is incorrect (for the related criticism see [25]).

In Fig. 4 we show how the unitarization affects the energy dependence of $d \sigma /\left.d t\right|_{t=0}$ for the real photoproduction of the $\rho^{0}$ and $J / \Psi$. In the former case the scanning radius is large, $r_{S} \gtrsim 1 \mathrm{f}$, in the latter case $r_{S} \sim 0.4 \mathrm{f}$. We also show the unitarization effect for the virtual photoproduction of the longitudinally polarized $\rho^{0}$ meson at $Q^{2}=120 \mathrm{GeV}^{2}$, appropriate for the scanning radius $r_{S} \sim r_{\Delta} \sim 0.15 \mathrm{f}$. Notice a rapid decrease of the unitarization correction with the decrease of the scanning radius $r_{S}$.

For a crude estimate of elastic production cross section we can take $B\left(\rho^{0}\right) \approx 10 \mathrm{GeV}^{2}$ [6] and $B(J / \Psi) \approx 4 \mathrm{GeV}^{2}[26]$. For the real photoproduction of the $\rho^{0}$ at $\sqrt{s}=\sqrt{2 m_{p} \nu}=$ $200 \mathrm{GeV}$, we find $\sigma\left(\gamma p \rightarrow \rho^{0} p\right) / \sigma_{\text {tot }}(\gamma p)=0.085$ (for the bare, non-unitarized, cross sections this ratio equals 0.135 ), in good agreement with the ZEUS determination $0.1 \pm 0.04$ [6]. For the real photoproduction of the $J / \Psi$ at $\nu=150 \mathrm{GeV}$ we find $\sigma(\gamma p \rightarrow J / \Psi p) \sim 16 \mathrm{nb}$, which agrees with the E687 result of $17.9 \pm 4.0 \mathrm{nb}$ at $\nu=177 \mathrm{GeV}$ and $9.8 \pm 2.9 \mathrm{nb}$ at $\nu=121 \mathrm{GeV}[26]$. The rise of the $J / \Psi$ production cross section with energy observed by E687 is also consistent with our prediction in Fig. 4. Our estimate for the real photoproduction of the $J / \Psi$ at HERA at $\sqrt{s}=200 \mathrm{GeV}$, i.e., $\xi=8.3$ is $\sigma_{t o t}(\gamma p \rightarrow J / \Psi p) \sim 90 \mathrm{nb}$, i.e., we predict the fivefold increase from FNAL to HERA. Theoretical calculations of the forward production cross section are more straightforward and are free of uncertainties with the energy dependence of 
the diffraction slope, and it is very much desirable that the experimental data are presented in the form of $d \sigma /\left.d t\right|_{t=0}$. More detailed comparison with the experiment will be presented elsewhere.

In Fig. 5 we present our predictions for the effective intercept

$$
\Delta_{e f f}\left(\xi, Q^{2}\right)=\frac{1}{2} \cdot \frac{\left.\left.\partial \log (d \sigma / d t)\right|_{t=0}\right)}{\partial \xi}
$$

for the transverse and longitudinal polarizations of the $J / \Psi$ and $\rho^{0}$. The rise of $\Delta_{e f f}\left(\xi, Q^{2}\right)$ with energy and $Q^{2}$ is an interplay of the BFKL perturbative and the nonperturbative cross section. On the other hand, for the $J / \Psi$ and the longitudinally polarized $\rho^{0}$, at $Q^{2} \approx$ $C^{2} / r_{\Delta}^{2} \sim(100-200) \mathrm{GeV}^{2}$ we predict the precocious BFKL behaviour $\Delta_{\text {eff }}\left(\xi, Q^{2}\right) \approx \Delta_{\mathbf{I P}}$. Such a behaviour of $\Delta_{e f f}\left(\xi, Q^{2}\right)$ is a very definitive prediction of our approach. Because of a large value of $C_{T}\left(\rho^{0}\right)$, in the transverse $\rho^{0}$ production the magic scanning radius $r_{S} \sim r_{\Delta}$ and $\Delta_{e f f}\left(\xi, Q^{2}\right) \approx \Delta_{\mathbb{P}}$ are not attainable even at $Q^{2} \sim 200 \mathrm{GeV}^{2}$. This prediction is difficult to test, though, because $\sigma_{T} \ll \sigma_{L}$. It is interesting that one has $r_{S} \sim r_{\Delta}$ also for the quasireal photoproduction of the $\Upsilon(1 S)$, which reaction can even be favoured for the higher flux of quasireal photons. For the $\rho^{0}$ and $J / \Psi$ production at large $Q^{2}$, the kinematical range of HERA corresponds to $\exp (-\xi) \approx x \gtrsim 10^{-5} \cdot\left(Q^{2} / \mathrm{GeV}^{2}\right)$, in which region the unitarization effects can still be neglected. We emphasize that precisely the same approach with the same dipole cross section, gives a very good description of the HERA data on $F_{2}\left(x, Q^{2}\right)[15]$.

Within our lightcone formalism, the dipole cross section is a universal quantity, and all the dependence on the process is contained in wave functions of the initial and secondary particle. The point we wish to make is that once the different production processes $\gamma^{*} p \rightarrow$ $V_{i} p$ are studied at values $Q_{i}^{2}$ so arranged as to have the same scanning radius $r_{S}$ Eq. (凹) , then the production cross sections will exhibit identical energy dependence. One must compare the cross sections at energy $\nu_{i}$ corresponding to the same rapidity $\xi$. For instance, we predict identical energy $(\xi)$ dependence of the real photoproduction of the $J / \Psi$ and of the virtual photoproduction of the longitudinal and transverse $\rho^{0}$ at $Q^{2} \approx 20 \mathrm{GeV}^{2}$ and $\approx 30 \mathrm{GeV}^{2}$, respectively. This rescaling from one vector meson to another constitutes an important cross-check of the whole formalism.

More predictions can be made, which are specific of the small gluon correlation radius 
$R_{c}=0.3 \mathrm{f}$. Namely for this reason, the perturbative BFKL cross section $\sigma^{(p t)}(\xi, r)$ is concentrated at smaller $r$ than the $\sigma^{(n p t)}(r)$, and the onset of the dominance of the BFKL cross section is followed by a change of the $Q^{2}$ dependence of the forward production cross section. In Fig. 6 we present our predictions for the energy dependence of

$D_{T}=\left.\left(m_{V}^{2}+Q^{2}\right)^{4} \cdot \frac{d \sigma_{T}}{d t}\right|_{t=0}, \quad D_{L}=\left.\left(m_{V}^{2}+Q^{2}\right)^{4} \cdot \frac{m_{V}^{2}}{Q^{2}} \cdot \frac{d \sigma_{L}}{d t}\right|_{t=0}, \quad R=\frac{m_{V}^{2}}{Q^{2}} \frac{d \sigma\left(\gamma_{L}^{*} \rightarrow V_{L}\right) L}{d \sigma\left(\gamma_{T}^{*} \rightarrow V_{T}\right)}$.

The gradual rise of $D_{L, T}$ with $Q^{2}$ comes predominantly from the rising density of gluons, see Eq. (9). Notice, that the ratio $d \sigma_{L} / d \sigma_{T}$ rises much slower than $Q^{2} / m_{V}^{2}$ and exhibits a substantial energy dependence at fixed $Q^{2}$, which can be tested at HERA. We strongly advocate studying the $Q^{2}$ dependence at fixed rapidity $\xi$ rather than the fixed energy $\nu$.

Eqs. (9-11) show that the vector-meson production amplitude measures the dipole cross section $\sigma\left(\xi, r_{S}\right)$ and the gluon structure function of the proton. We wish to emphasize that this measurement is exceedingly sensitive to the $r_{S}-Q^{2}$ relationship. Typically, one will probe the gluon structure function $G\left(\xi, Q^{2}\right)$ at the factorization scale $q^{2} \approx \tau\left(Q^{2}+m_{V}^{2}\right)$, where

$$
\tau_{T, L} \approx \frac{A}{C_{T, L}^{2}}
$$

In the range of $Q^{2} \sim(10-100) \mathrm{GeV}^{2}$ of the practical interest, we find $\tau_{T, L}(J / \Psi) \sim 0.2$, $\tau_{L}\left(\rho^{0}\right) \sim 0.15$ and $\tau_{T}\left(\rho^{0}\right) \sim(0.07-0.1)$. The emergence of such a dramatically small rescaling coefficients $\tau_{T, L}$ is a consequence of colour transparency. It was overlooked in [27].

A brief commment on the production of the $2 S$ radial excitations $\rho^{\prime}$ and $\Psi^{\prime}$ is in order. The wave function of the $2 S$ state has a node, because of which the corresponding weight function $W_{T, L}\left(Q^{2}, r^{2}\right)$ changes the sign at $r \sim R_{V}$. The resulting cancellations of the $r>R_{V}$ and $r<R_{V}$ contributions to the production amplitude (the node effect) lead to a strong suppression of the $\rho^{\prime} / \rho^{0}$ and $\Psi^{\prime} /(J / \Psi)$ production ratio [7-11]. With increasing energy and with the onset of the dominance of $\sigma^{(p t)}(\xi, r)$, which is concentrated at smaller $r$, the node effect will decrease. For instance, in the real photoproduction the $\Psi^{\prime} /(J / \Psi)$ ratio is expected to increase by the factor $\sim 3$ from the CERN/FNAL energies to the highest energies available at HERA [28]. The interesting possibility [28] is that for the radially excited light mesons $\left(\phi^{\prime}, \omega^{\prime}, \rho^{\prime}\right)$, in the real photoproduction the $V^{\prime} / V$ ratio may exhibit the anomalous, nonmonotonic energy dependence, when this ratio first decreases with energy, 
and then starts increasing. In the opposite to this, at large $Q^{2}$ such that $r_{S} \ll R_{V}$, the node effect disappears, and we expect $\rho^{\prime} / \rho^{0} \sim \Psi^{\prime} /(J / \Psi) \sim 1$ over the whole energy range.

\section{Conclusions:}

The purpose of this paper was to examine how the dipole cross section $\sigma(\xi, r)$ is scanned in the virtual photoproduction of vector mesons. The BFKL dipole cross section, complemented by the nonperturbative dipole cross section at large $r$, gives the unified description of the photoproduction processes in the whole range of $\nu$ and $Q^{2}$, including the real photoproduction. The same dipole cross section gives a good description [14] of the proton structure function measured at HERA. Changing $Q^{2}$, one can probe the energy dependence of $\sigma(\xi, r)$ in a broad range of radii from the nonperturbative region of $r \sim 1 \mathrm{f}$ down to the perturbative domain of $r \ll 1$. One can zoom at the magic radius $r_{\Delta}$ and determine at HERA the pomeron intercept $\Delta_{\mathbb{I}}$, measuring the cross section of elastic $\rho^{0}, J / \Psi$ production at at $Q^{2} \sim(100-200) \mathrm{GeV}^{2}$ and the quasireal photoproduction of the $\Upsilon(1 S)$. We argued that this determination of $\Delta_{\mathbb{P}}$ is not affected by the unitarity corrections. We make a strong point that it is the dipole size $r$ rather than the photon's virtuality $Q^{2}$, which controls the energy dependence of diffractive amplitudes: the value of $Q^{2}$ needed to scan the dipole cross section at the magic radius $r_{\Delta}$ strongly varies from the process to process, c.f. the discussion in [15]. We predict very specific variation with energy of the $Q^{2}$ dependence of elastic production cross section and of the $L / T$ ratio, which derive from the small correlation radius for perturbative gluons $R_{c} \sim 0.3 \mathrm{f}$. Elastic production is found to probe the gluon structure function of the proton at an anomalously small factorization scale. 


\section{References}

[1] E.A.Kuraev, L.N.Lipatov and V.S.Fadin, Sov.Phys. JETP 44 (1976) 443; 45 (1977) 199; Ya.Ya.Balitskii and L.N.Lipatov, Sov. J. Nucl. Phys. 28 (1978) 822; L.N.Lipatov, Sov. Phys. JETP 63 (1986) 904; L.N.Lipatov. Pomeron in Quantum Chromodynamics. In: Perturbative Quantum Chromodynamics, editor A.H.Mueller, World Scientific, 1989.

[2] N.Nikolaev and B.G.Zakharov, The triple-pomeron regime and the structure function of the pomeron in the diffractive deep inelastic scattering at very small $x$, Landau Inst. preprint Landau-16/93 and Jülich preprint KFA-IKP(Th)-1993-17, June 1993, to appear in Z. Phys. C (1994); The pomeron in the diffractive deep inelastic scattering, to appear in JETP 78(5) (1994).

[3] N.N.Nikolaev, B.G.Zakharov and V.R.Zoller, JETP Letters 59 (1994) 8;

[4] N.N.Nikolaev, B.G.Zakharov and V.R.Zoller, The BFKL and GLDAP regimes for the perturbative QCD pomeron, Jülich preprint KFA-IKP(TH)-1994-02, January 1994; JETP 78(6) (1994).

[5] M.Derrick et al., ZEUS Collaboration, Phys. Lett. B (1993); I.Abt et al., H1 Collaboration, Nucl. Phys. B407 (1993) 515.

[6] ZEUS Collaboration: M.Derrick et al., DESY 94-032 (1994); Phys. Lett. B293 (1992) 465; H1 Collaboration: T.Ahmed et al., Phys. Lett. B299 (1993) 374.

[7] N.N.Nikolaev, Comments on Nucl. Part. Phys. 21 (1992) 41.

[8] B.Z.Kopeliovich and B.G.Zakharov, Phys. Rev. D44 (1991) 3466;

[9] O.Benhar, B.Z.Kopeliovich, C.Mariotti, N.N.Nikolaev and B.G.Zakharov, Phys. Rev. Lett. 69 (1992) 1156 
[10] B.Z.Kopeliovich, J.Nemchik, N.N.Nikolaev and B.G.Zakharov, Phys. Lett. B309 (1993) 179.

[11] B.Z.Kopeliovich, J.Nemchik, N.N.Nikolaev and B.G.Zakharov, Phys.Lett. B324 (1994) 469.

[12] N.N.Nikolaev, B.G.Zakharov and V.R.Zoller, The spectrum and solutions of the generalized BFKL equation for total cross sections, Jülich preprint KFA-IKP(TH)-199401, January 1994, to appear in Phys. Lett. B (1994);

[13] N.N.Nikolaev and B.G.Zakharov, The BFKL evolution and universal structure function at very small $x$, Jülich preprint KFA-IKP(TH)-1994-05, January 1994; to appear in Phys. Lett. B (1994);

[14] N.N.Nikolaev and B.G.Zakharov, Deep inelastic scattering at HERA and the BFKL pomeron, Jülich preprint KFA-IKP(TH)-1993-06, January 1994, to appear in Phys. Lett. B (1994).

[15] N.N.Nikolaev and B.G.Zakharov, How to measure the intercept of the BFKL pomeron at HERA, Jülich preprint KFA-IKP(TH)-1994-10, February 1994, to appear in Phys. Lett. B (1994).

[16] J.Nemchik, N.N.Nikolaev and B.G.Zakharov, paper in preparation.

[17] N.N. Nikolaev and B.G. Zakharov, Z. Phys. C49 (1991) 607; Z. Phys. C53 (1992) 331;

[18] V.Barone, M.Genovese, N.N.Nikolaev, E.Predazzi and B.G.Zakharov, Z. Phys. C58 (1993) 541; Int.J.Mod.Phys A, 8 (1993) 2779.

[19] V.Barone, M.Genovese, N.N.Nikolaev, E.Predazzi and B.G.Zakharov, Phys.Lett. B326 (1994) 161.

[20] V.Barone, M.Genovese, N.N.Nikolaev, E.Predazzi and B.G.Zakharov, Phys.Lett. B304 (1994) 176.

[21] D.O.Caldwell et al., Phys. Rev. Lett. 40 (1978) 1222. 
[22] A.Donnachie and P.V.L.Landshoff, Phys. Lett. B296 (1992) 227.

[23] B.Z.Kopeliovich, N.N.Nikolaev and I.K.Potashnikova, Phys. Rev D39 (1989) 769.

[24] J.R.Forshaw and J.K.Storrow, Phys. Lett. B321 (1994) 151.

[25] N.N.Nikolaev, Elastic and Diffractive Scattering: 3-d Blois Workshop, Nucl. Phys. B (Proc. Suppl.) 12 (1990) 93.

[26] P.L.Frabetti et al., Phys. Lett. B316 (1993) 197.

[27] S.J.Brodsky et al., SLAC-PUB-6412R (1994).

[28] N.N.Nikolaev and B.G.Zakharov, paper in preparation. 


\section{Figure captions:}

Fig. 1 - Weight functions $W_{T, L}\left(Q^{2}, r^{2}\right)$ as a function of a variable $y=r^{2}\left(Q^{2}+m_{V}^{2}\right)$.

Fig. 2 - Decomposition of the dipole cross section into the BFKL perturbative $\sigma^{(p t)}(\xi, r)$ and the nonperturbative $\sigma^{(n p t)}(r)$ components. The growth of the BFKL cross section with energy (rapidity) is shown.

Fig. 3 - The predicted energy dependence of the (B) bare and (U) unitarized total $\rho^{0} p$ and real photoabsorption cross section. The data shown are from the HERA [6] and FNAL [21] experiments.

Fig. 4 - The predicted energy (rapidity) dependence of the (B) bare and (U) unitarized differential cross section of forward real and virtual photoproduction of the $\rho^{0}$ and $J / \Psi$.

Fig. 5 - The energy (rapidity) and $Q^{2}$ dependence of the effective intercept $\Delta_{e f f}\left(\xi, Q^{2}\right)$ for the forward production of the transverse and longitudinal $\rho^{0}$ and $J / \Psi$.

Fig. 6 - Predictions for the $Q^{2}$ dependence of the differential cross section of forward production and the ratio of the longitudinal and transverse cross sections. Shown are the quantities $D_{T, L}$ and $R$ defined in the text. 

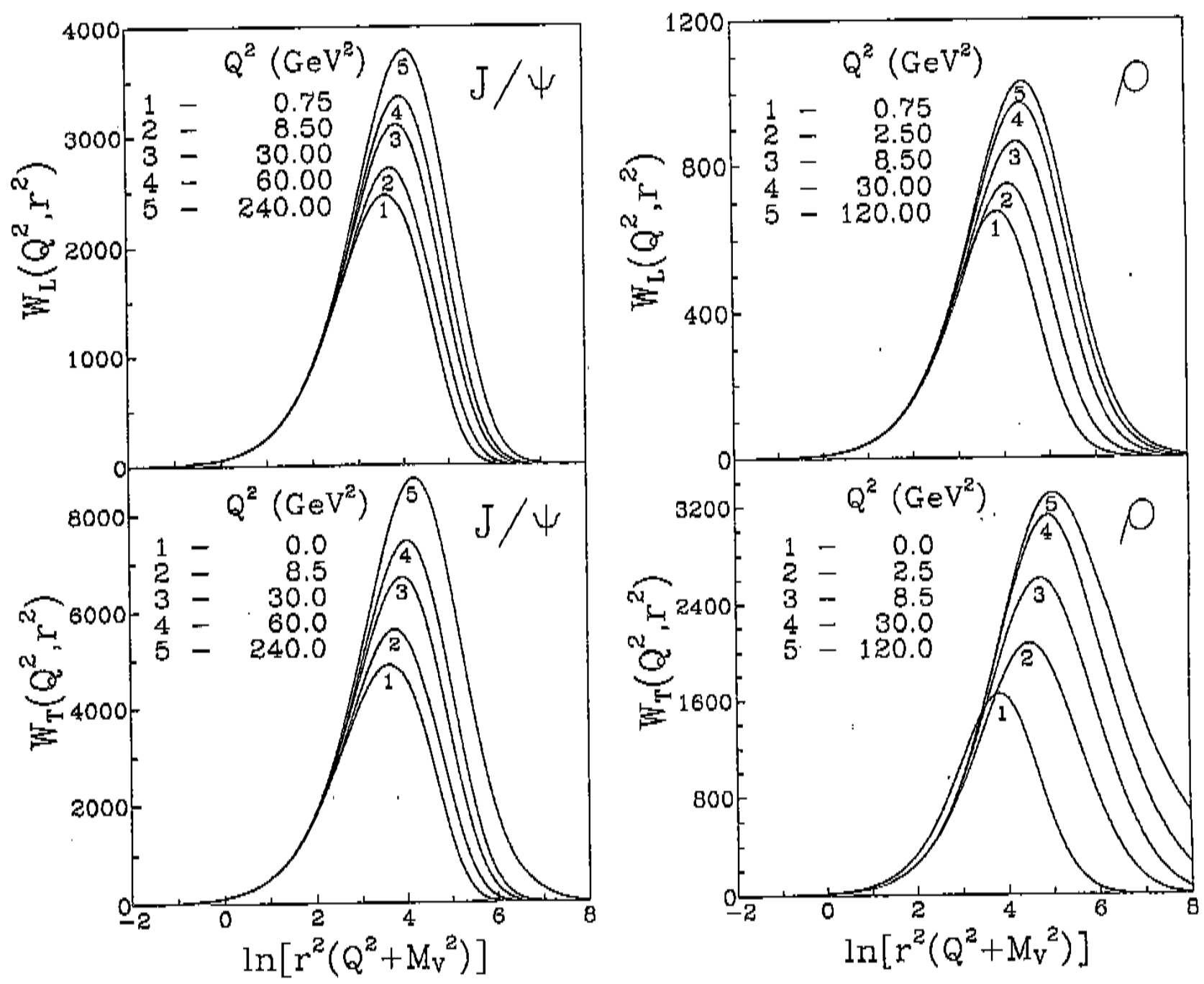

Fig. 1 


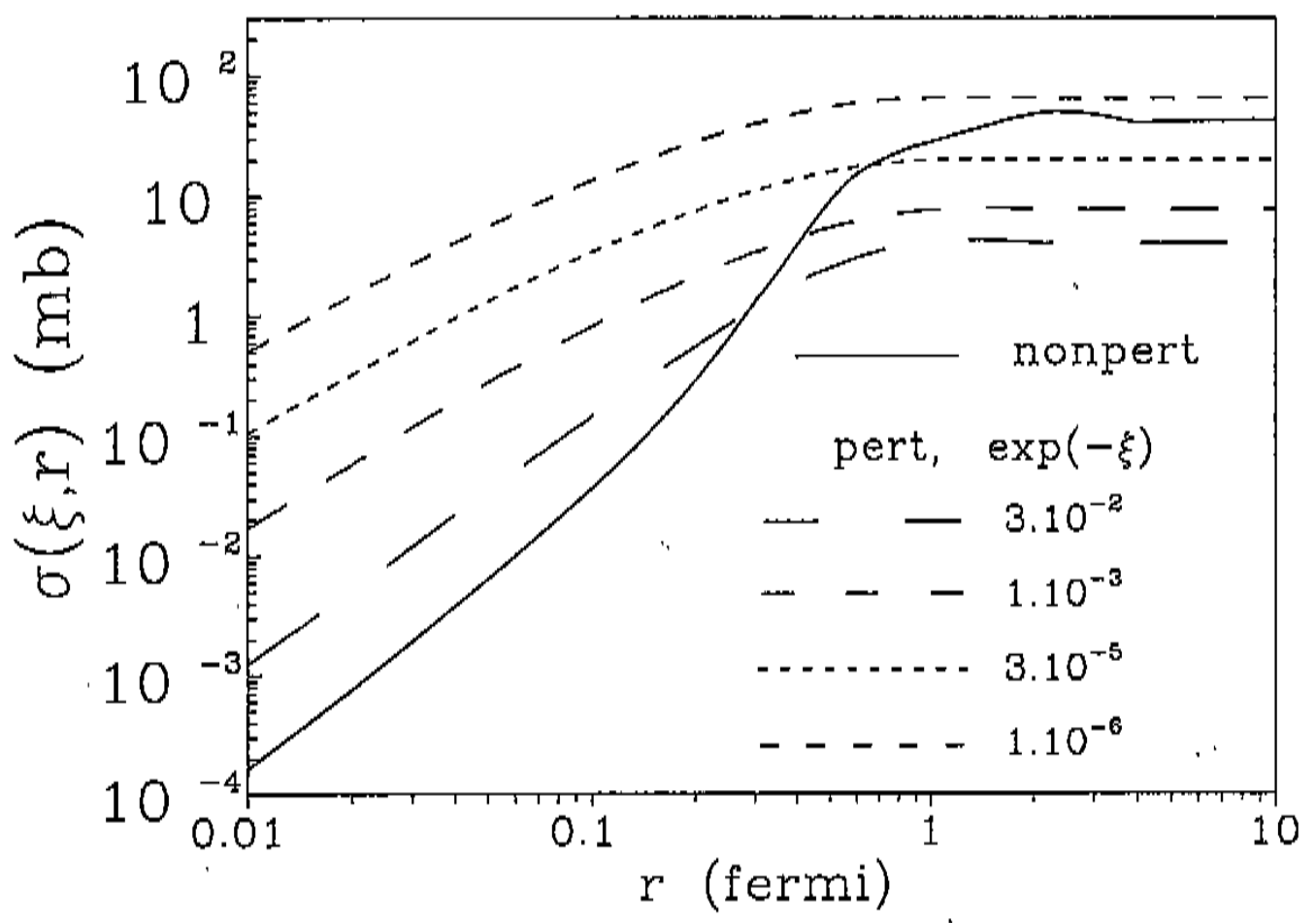

Fig. 2 


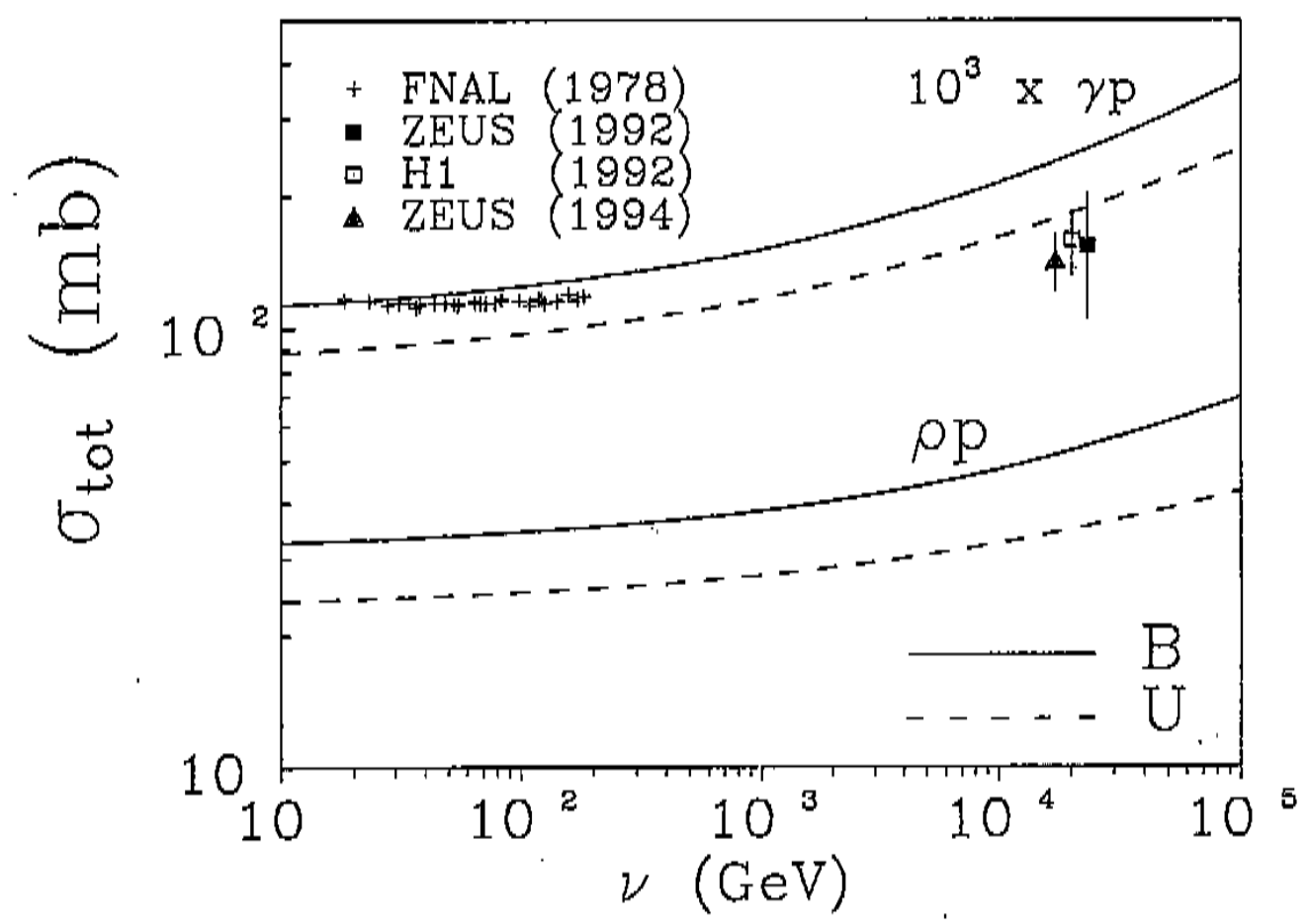

Fig. 3 


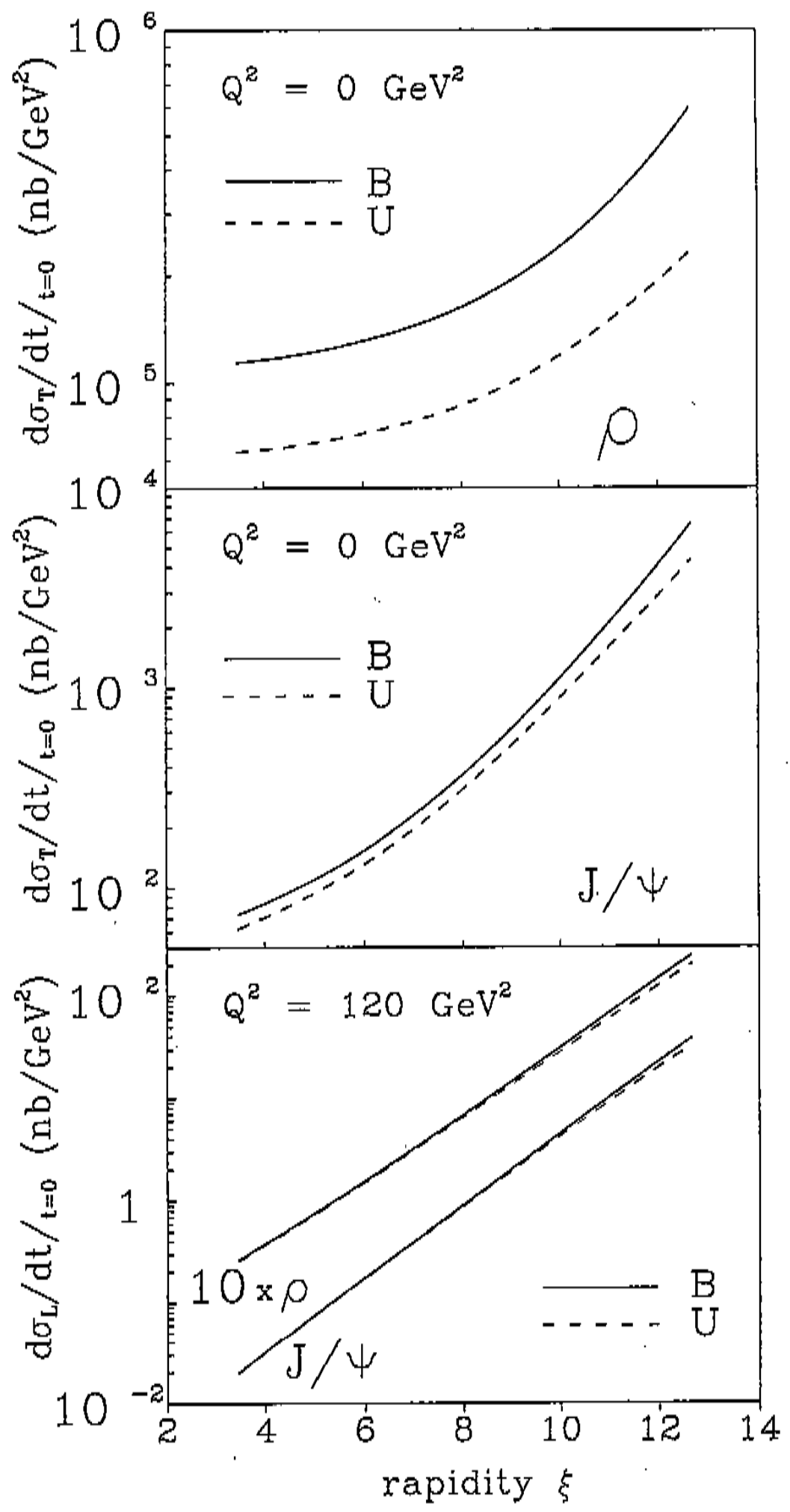

Fig. 4 


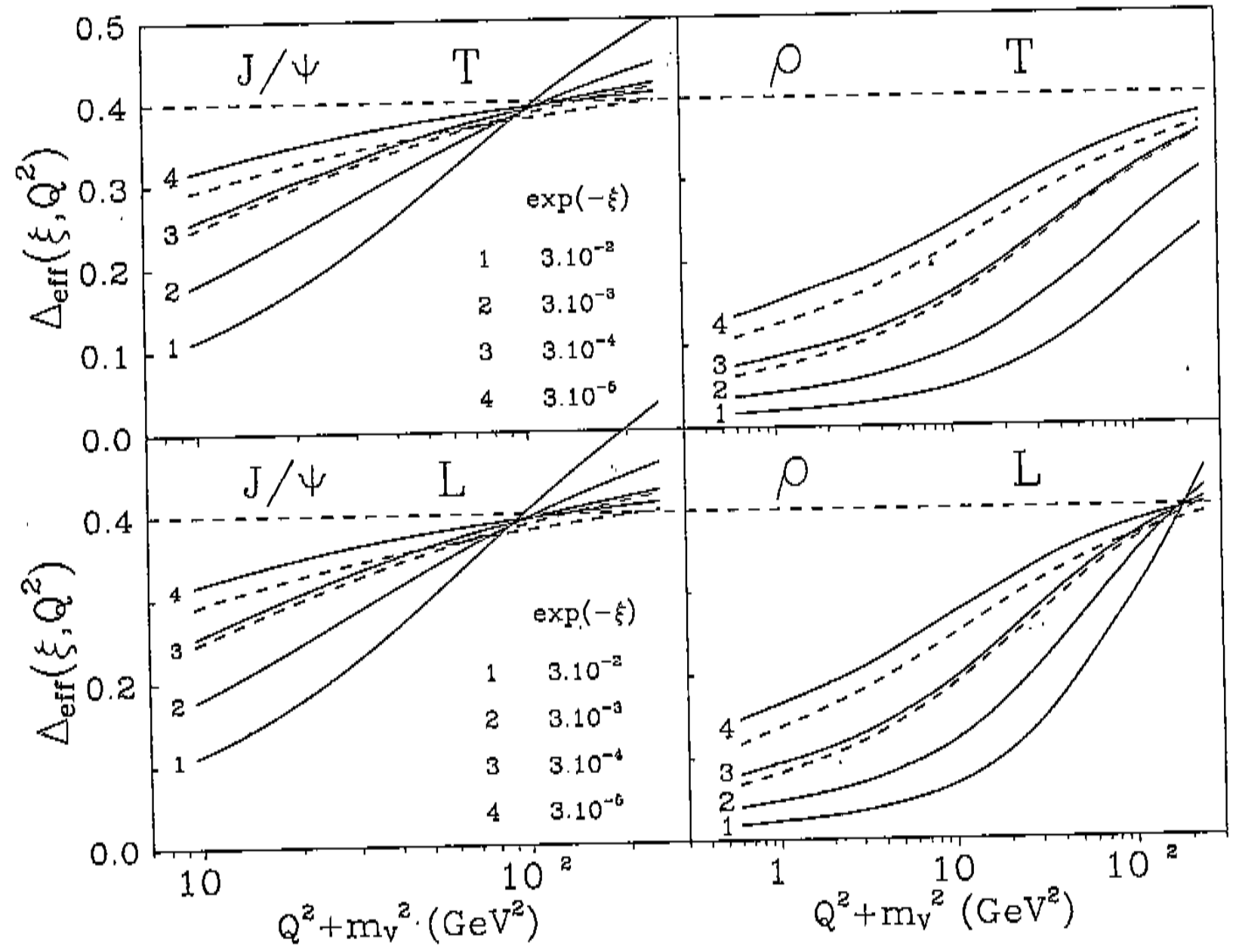

Fig. 5 

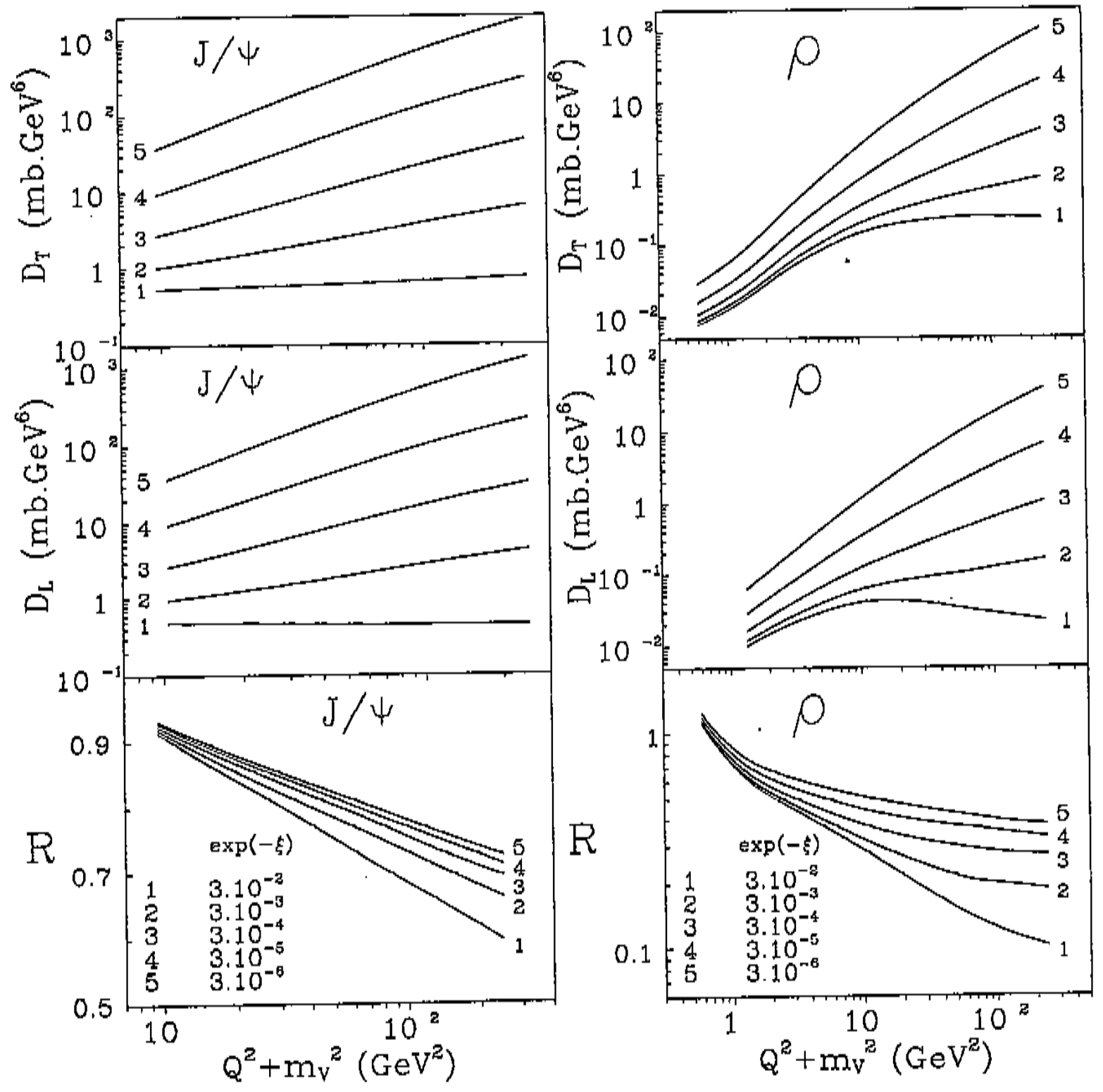

Fig. 6 